\title{
A Novel Routing Protocol Based on the Channel Load Similarity for Multi-interface Wireless Mesh Networks
}

\author{
Tian Qiming ${ }^{1, a}$ \\ ${ }^{1}$ Dept. of Computer, Wenzhou Vocational \& Technical College, Wenzhou 325035, China \\ a'Tqm78@126.com
}

Keywords: routing metric; wireless mesh networks; channel load similarity; link load

\begin{abstract}
Routing protocols are the key factors which determine the communication performance in the multi-interface wireless mesh networks. The existing routing protocols can not capture the channel interference accurately. In order to utilize the multi-interface technology to reduce the interference and improve the network throughput, this paper presented a novel routing metric for multi-interface wireless mesh networks. This metric integrated both the channel similarity and the link load to measure the route quality. Furthermore, this paper applied this routing metric to the AODV routing protocol which formed a new routing protocol. The simulation results show that the new protocol outperforms the original AODV protocol in terms of the average throughput, transmission rate and end-to-end delay.
\end{abstract}

\section{Introduction}

With the advantages of flexible structure, easy installment, low prepaid cost, robustness and so on, the wireless mesh network (WMN) becomes a key technology of new broadband wireless access, completely different from traditional wireless network. However, as in a single-interface wireless mesh network, all links are working on the same interface, interference among channels are serious. In order to satisfy the rising capacity demand of WMN's application in real life, multi-interface technology is used in the WMN to reduce link interference. A router in the multi-interface WMN has several wireless interfaces, working on orthogonal channels to greatly reduce interference among channels. Therefore, it has become a hotspot in today's research how to employ the multi-interface technology in designing routing metric in order to effectively reduce interference and raise throughput.

\section{Relevant Work}

Routing Metric. For routing protocols of multi-interface wireless mesh network, design of routing metric is the key factor that affects network performance. In recent years, some routing metrics used in WMN have been raised, such as ETX [1], ETT [2], WCETT [2], MIC [3] and so on.

Expected Transmission Count. The expected transmission count (ETX) is defined as the expected transmission number that the MAC layer needs to successfully deliver a data packet through a wireless link. It inaccurately evaluates the transmission number of uniscast packets by testing the packet loss rate of broadcast packets among nodes. Moreover, ETX does not consider the channel interference.

Expected Transmission Time. The expected transmission time (ETT) is defined as the accumulated value of expected transmission time that the MAC layer needs to successfully transmit a data packet though a wireless link. ETT considers the influence of transmission speed on the basis of ETX. ETT captures the influence of link capacity on path performance, but does not consider the channel interference.

Weighted Cumulative Expected Transmission Time. The weighted cumulative expected transmission time (WCETT) comprehensively considers packet loss rate, bandwidth and other link performance parameters. Based on ETT, WCETT reduces channel interference by reducing the number of nodes which transmit data on the same channel. Though WCETT can get a balance between network throughput and delay, it roughly evaluates channel interference of the whole routine, not paying attention to the interference degree among neighboring channels and the influence of link load on channel interference. 
Metric of Interference and Channel Switching. The metric of interference and channel switching (MIC) makes some improvements based on WCETT. MIC more accurately considers the interference degree among neighboring channels and surrounding nodes. But MIC just simply considers the channel similarity within the adjacent jumps, but not the adjacent two jumps. Moreover, MIC does not consider the influence of link load on channel interference.

AODV Protocol. The AODV protocol is a classic demand routing algorithm, which means that, when the original node needs to communicate with some objective node, it establishes a routing path between nodes. Routing information is stored with some certain TTL, which will not be stored all the time. If a routing path is no longer needed, it will be deleted. Its procession is short and its storage cost is small, able to efficiently use bandwidth and make quick response on changes of network topology. However, as AODV protocol uses jumps as its routing metric, it chooses a routine to deliver data packets with the least jumps from the original node to the objective node, not considering any other link features. Therefore, it is weak to resist data flow interference.

\section{Routing Protocol based on Channel Load Similarity}

Channel Load Similarity. In the multi-interface wireless Mesh network, if several links are working on the same channel, they will compete for channel resources, thus to create channel interference. If several links are working on the orthogonal channels, channel interference among links is greatly reduced. Therefore, in choosing routing path, it is necessary to consider the channel differentiation. Assuming a routine has $N$ jumps, a link with $N a$ jumps is working on 802.11a channel, and another link with $\mathrm{Ng}$ jumps is working on $802.11 \mathrm{~g}$ channel, the channel differentiation of the routine is defines as:

$$
C D I=\frac{\min (N a, N g)}{2 \times\lfloor N / 2\rfloor}
$$

From Formula (1), a channel differentiation definition for a routine working on several orthogonal channels can be deduced. Assuming all links can choose $c$ channels on a routing $p$, these channels are orthogonal, total jump number of $p$ is $N$, total jump number on channel $c$ is $N c$, the channel differentiation on routine $p$ is as follows:

$$
C D I_{p}=\frac{\min \left(N_{1}, N_{2}, \cdots, N_{c}\right)}{c \times\lfloor N / c\rfloor}
$$

The definition only simply shows the overall difference degree of the channels, but does not consider that the difference degree of neighboring channels affects channel interference more greatly than the difference degree of non-neighboring channels. In fact, due to the exposed terminal problems, if links within two neighboring jumps are working on the same channel, they will produce larger interference than within the non-neighboring two jumps [4]. Therefore, for Routine 1 and 2 in Figure 1, Formula (2) suggests that the two routines have the same channel difference degree. But, in fact, interference produced by Routine 1 is larger than that produced by Routine 2 .

In order to improve the defect of $C D I$, we introduce a new parameter: $S$ (ci,cj) to describe the channel similarity between the neighboring link $i$ and $j$. Definition is shown in Formula (3). In it, $c i$ and $c j$ respectively show the channels of the two neighboring links.

$$
S\left(c_{i}, c_{j}\right)= \begin{cases}a & c_{i}=c_{j} \\ b & c_{i} \neq c_{j}\end{cases}
$$




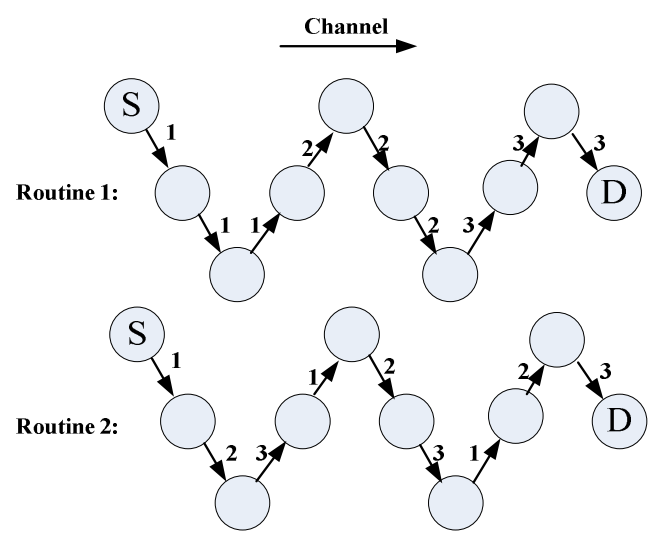

Figure 1 Comparison of Channel difference Degrees on the Two Routines

Obviously, the larger channel similarity is, the larger interference degree among channels will be. As the neighboring links produce more interference when working on the same channel than working on different channels, the channel similarity $a>>b$. Here, we make $a=1, b=0.001$.

Due to the exposed terminal problem, interference always occurs within the neighboring two jumps. In order to better characterize interference among channels, we improve Formula (2) with channel similarity parameter $S(c i, c j)$ and get Formula (4). Assuming that in Routine $p, c i$ and $c j$ respectively show the channels of the neighboring two links, and the total jump number of Routine $p$ is $N$, the formula is defined as follows.

$$
C S I_{p}=\sum_{i=1}^{N-1} \sum_{j=i+1}^{\min (i+2, N)} S\left(c_{i}, c_{j}\right)
$$

From Formula (4), CSI of the two routines in Figure 1 is as follows:

$$
\begin{aligned}
& C S I_{p 1}=9 \times 1+6 \times 0.001=9.006 \\
& C S I_{p 2}=15 \times 0.001=0.015
\end{aligned}
$$

From Formula (5) and (6), we know that CSI of Routine 1 is larger than that of Routine 2, which indicates that interference produced by Routine 1 is larger than that produced by Routine 2 .

However, channel interference is not only related to channel similarity, but also with the link load on the channel. If data packets choose the large-load channels to transmit information, it is possible that transmission delay will be prolonged due to collisions and blocks of data packets, even the loss of data packets.

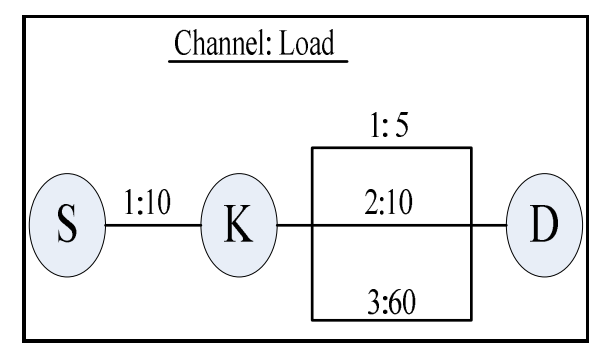

Figure 2 Routing Choice Considering the Load

In Figure 2, if Channel 1 is adopted in the links from Node $S$ to $K$, and the load is 10 . When choosing the routing link from Node $K$ to $D$, we should not only consider the channel similarity, but also the link load. If the router from Node $K$ to $D$ chooses the link on Channel 3, blocks of data packets may be possible due to the large link load. Therefore, it's better for the link from Node $K$ to $D$ to choose Channel 2 than Channel 3. After generally considering channel similarity and link load, we define the channel load similarity on Routine $p$ as follows. 


$$
C L S_{p}=\sum_{i=1}^{N-1}\left(\frac{Q L_{i}}{Q L_{\max }}+\sum_{j=i+1}^{\min (i+2, N)} S\left(c_{i}, c_{j}\right) \cdot \frac{Q L_{j}}{Q L_{\max }}\right)
$$

In it, $N$ is the total jump number of Routine $p$, and $Q L i$ and $Q L j$ respectively show the load in link $i$ and $j$ within the neighboring two jumps. The link load is represented by the average buffer queue length (i.e. the average number of packets in the buffer) when the data packet is flowing on the link of Routine $p$. QLmax represents the max buffer queue length on the link. Obviously, CLSp is composed with 2 parts: The first part is about the load of link $i$, and the second part is about the load interference produced by link $j$ within two jumps from link $i$. From Formula (7), the larger $Q L$ is, the smaller QLmax is, the larger $S(c i, c j)$ is, the higher channel interference degree will be.

Routing Protocol based on Channel Load Similarity. For the weak capacity of AODV protocol's resistance against channel interference, we apply channel load similarity as routing metric into AODV protocol. Thus, a new routing protocol CLSR is formed.

CLSR is different from AODV protocol in routing discovery period. In CLSR protocol, each intermediate node adds their number, channel number, link load and other information before forwarding a routing request. When the routing request reaches the objective node, the value of CLS metric can be calculated according to information in routing request packet, and a reverse route with the smallest cost will be chosen. A route reply packet with all member values of CLS metrics will be sent to the original node along the chosen reverse route. Each forwarding node in route reply updates the member value related to the link condition in CLS metric, such as the link load and so on. When the original node receives the reply packet, value of CLS metric will be updated according to the information in reply packet. By comparing the metric of the path carried by the reply packet with that in the routing table, the original node chooses a routine with the minimum CLS cost to send data packets.

\section{Simulation}

In order to evaluate performance of CLSR routing metric, we compare the performances of the CLSR protocol with the original AODV protocol by using the NS2 simulation tool.

Simulation Realization of CLSR Protocol. The greatest difference between CLSR protocol and AODV protocol is that the former uses CLS routing metric, while the latter uses the jump number as the metric. In the routing discovery period of CLSR protocol, when the original node and the objective node receives and sends data packets, they should calculate and judge the routing metric. Among them, the load, node number and the adopted channel number are collected in calculating CLS routing metric when each data packet flows though the link before they are packed in the data package.

Set of Simulation Scene. The simulation scene is set by the TCL language programming. Simulated topology is made up with 25 static mesh nodes within 1000 square meters, shown in Figure 3 . Size of the data packet is 512 Byte, with $1 \mathrm{mb} / \mathrm{s}$ emission rate and 100 meters of transmission area. Each node has the same number of RF interfaces and the same channel distribution strategy. Each interface is configured to one orthogonal channel. Eight random interfered CBR data flows transmit the information at the same time. In order to study the capacity of routing protocols to utilize the multi-interface technology to reduce the interference, we study the behaviors of the AODV protocol and the CLSR protocol in the variation of the number of interfaces. In simulation process, we set 1 to 4 interface(s), and observe the performance changes of end-to-end delay, transmission rate of data packet and the average throughput.

Simulation Result. Figure 4, 5 and 6 show that the average end-to-end delay, transmission rate of data packet and the average throughput of the CLSR protocol are better than the AODV protocol. As CLSR considers the channel interference caused by channel similarity and load, it can better use the multi-interface and multi-interface capacity of the wireless mesh network to balance the node flow load of the whole network, so that it avoids the re-transmission or packet loss caused by collisions of the data packets in highly-competitive area. 

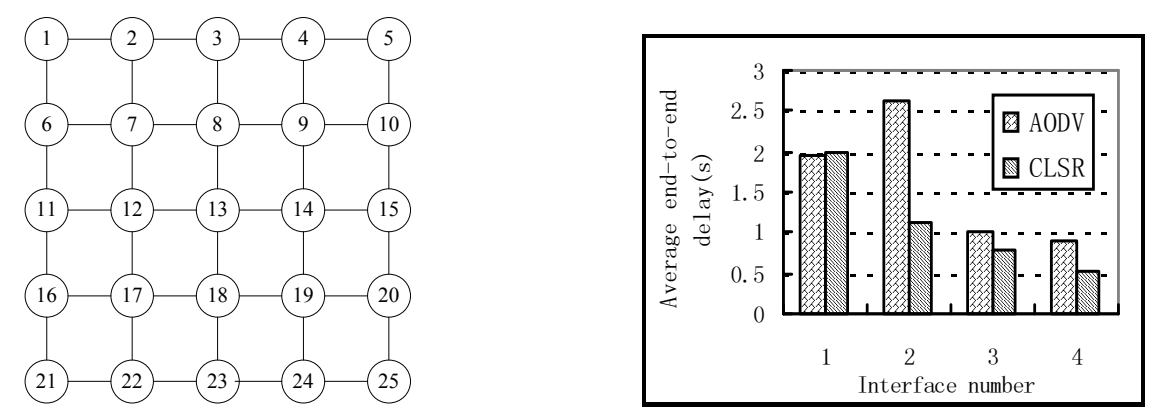

Figure 3 Simulation Topology Structure Figure 4 Average End-to-End Delay VS Interface Number

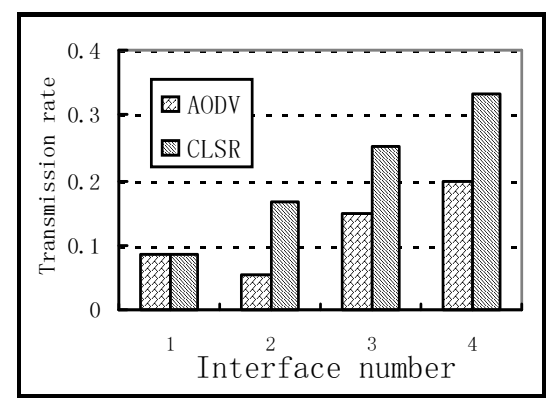

Figure 5 Transmission Rate of Data Packet VS Interface Number

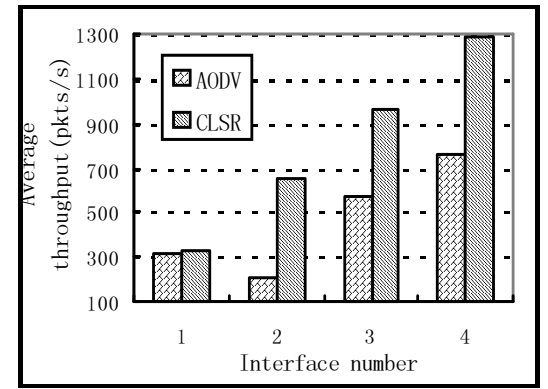

Figure 6 Average Throughput VS Interface Number

\section{Summary}

On some defects of the existing routing metrics, the essay comes up with a new routing strategy based on channel load similarity. The strategy comprehensively considers the channel similarity and link load within the neighboring two jumps. On this basis, the essay makes some improvements on AODV protocol, and raises a new routing protocol, CLSR, which makes up the defects that AODV protocol can not fully use the multi-interface technology to reduce interference. Simulation results show that CLSR routing protocol can fully make use of the multi-interface capacity of the nodes to choose a route with small interference, delay and packet loss rate.

\section{Acknowledgment}

This work was supported in part by the Educational Commission of Zhejiang Province (Y201122696).

\section{References}

[1] Douglas S. J. De Couto, Daniel Aguayo, John Bicket, Robert Morris. A high-throughput path metric for multi-hop wireless routing[C]. ACM Mobicom, 2003.

[2] DRAVES R, PADHYE J, ZILL B. Routing in multi-radio, multi-hop wireless mesh networks [C]. Philadelphia, PA USA: Proc of SIGMOBILE, 2004:114-128.

[3] YANG Y, WANG J, KRAVETS R. Interference-aware load balancing for multihop wireless networks [D].Urbana, IL USA: University of Illinois at Urbana-Champaign, 2005.

[4] Tian Qiming. A new interference-delay aware routing metric for multi-interface wireless mesh networks [C]. Chengdu, China: Proc of WICOM. 2010. 